\title{
Probing AGN Broad Line Regions with LAT Observations of FSRQs
}

\author{
Jennifer E. Carson*, James Chiang ${ }^{\dagger, * *}$ and Markus Böttcher \\ ${ }^{*}$ SLAC, 2575 Sand Hill Road, MS 29, Menlo Park, CA 94025 \\ ${ }^{\dagger}$ CRESST/UMBC \\ ** SLAC, 2575 Sand Hill Rd, Menlo Park CA 94025 \\ $¥$ Ohio University, Dept. of Physics \& Astronomy, Clippinger 339, Athens, OH 45701
}

\begin{abstract}
.
The GLAST Large Area Telescope (LAT) is expected to detect gamma-ray emission from over a thousand active galaxies[1,2], many of which will be flat spectrum radio quasars (FSRQs). A commonly assumed ingredient of leptonic models of FRSQs is the contribution to the gamma-ray flux from external inverse-Compton (EIC) scattering of photons from the broad line region (BLR) material by relativistic electrons and positrons in the jet. Here we explore the effect of the BLR geometry on the high-energy emission from FSRQs.
\end{abstract}

PACS: $98.54 . \mathrm{Cm}$

\section{THE MODEL}

The model of Böttcher et al. (1997)[3] and Böttcher \& Bloom (2000)[4] form the basis for this analysis. In this model (see Figure 1), pair plasma blobs are instantaneously injected into a cylindrical jet structure with bulk relativistic motion. The electrons have an initial power-law distribution over a range of energies $\left(\gamma_{\min }, \gamma_{\max }\right)$. A spherical shell of material of Thomson depth $\tau$ represents the BLR. The following radiation processes are included: synchrotron emission (Sy), inverse-Compton scattering of synchrotron photons (SSC), inverse-Compton scattering of radiation from the accretion disk entering the jet directly (ECD), and inverse-Compton scattering of accretion disk radiation scattered off broad-line region clouds (ECC). The particle distributions are evolved self-consistently as the blob cools.
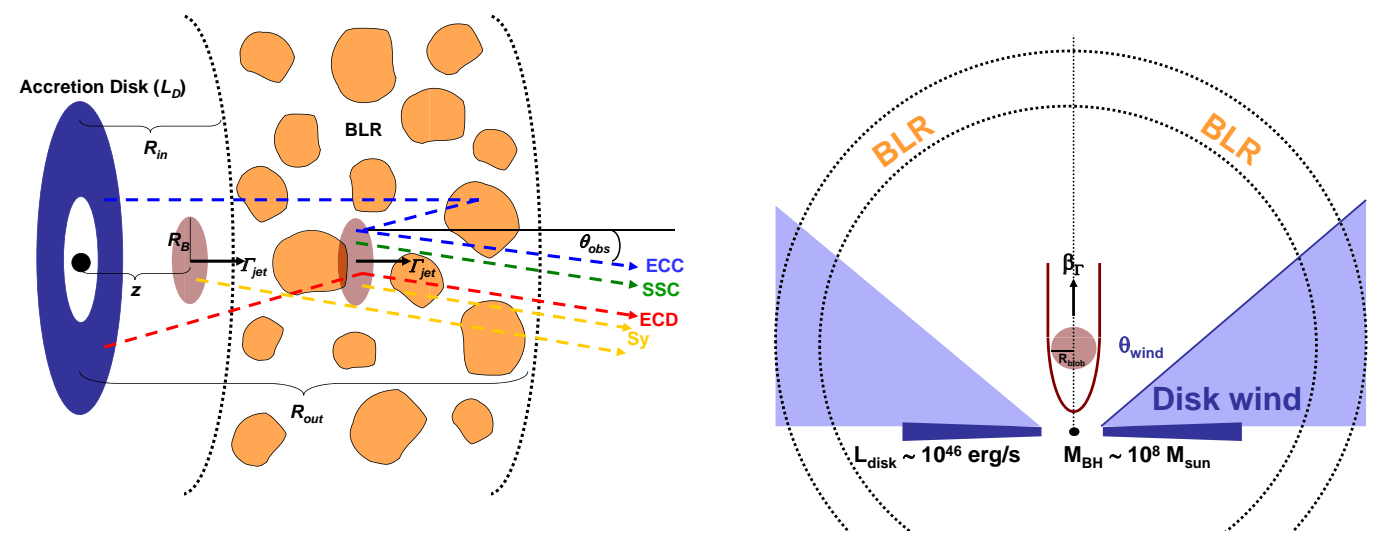

FIGURE 1. Cartoons of the leptonic emission model used, with radiation contributed from synchtron (Sy), synchrotron selfCompton (SSC), and external inverse-Compton emission (ECC, ECD). Left: A small-scale view, in which the jet blob is ejected to the right from the central region. Right: A large-scale view, in which the jet blob is ejected up away from the black hole; both broad line region geometries are depicted. 

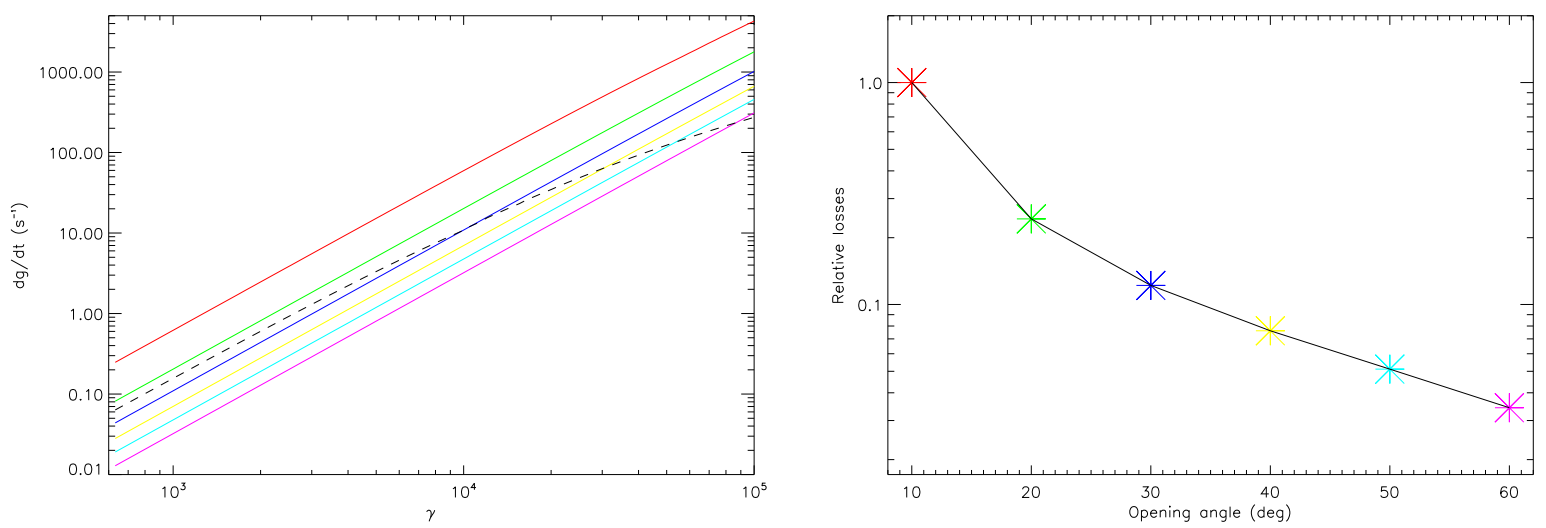

FIGURE 2. Left: The single-electron energy loss rate due to ECC scattering vs. electron energy $\gamma$ for opening angles $\theta_{\text {wind }}$ between $10^{\circ}$ and $60^{\circ}$ in $10^{\circ}$ increments. The dashed line shows the ECC losses for a standard spherical BLR with inner and outer radii of 0.1 and $0.4 \mathrm{pc}$, respectively, and an optical depth $\tau=0.1$. Right: The relative single electron energy loss ratios due to the disk wind as a function of $\theta_{\text {wind }}$.

\section{THE SIGNATURE OF THE BLR GEOMETRY IN THE LAT SED}

Past modeling of the ECC emission has considered only a fairly simple geometry for the BLR material: Thomson or resonant line scattering gas that is spherically distributed about the central black hole. However, optical and UV observations of Seyfert galaxies and radio-quiet quasars, as well as theoretical models, suggest that the BLR material may have a very different geometry, such as an equatorial disk wind[5]. To test this hypothesis, we have modified the electron cooling code developed by M. Böttcher to generalize the BLR geometry. We can model the disk wind by introducing an angular dependence $\left(\theta_{\text {wind }}\right)$ into the ECC calculations and evacuating a conical area around the jet (see Figure 1, right). The geometry of the remaining region, with $\theta_{\text {wind }}>\sim 30^{\circ}$, is similar to a wind geometry. We also set the inner radius to $100 R_{g}\left(7 \times 10^{-6} \mathrm{pc}\right.$ for a black hole mass of $\left.1.5 \times 10^{8} M_{\odot}\right)$, adopt $\theta_{\text {wind }}=0.5$, and introduce a density gradient $n \propto r^{-2}$.

We find:

1. The electron energy losses in the disk wind case are comparable to the electron losses in the spherical BLR case - that is, the disk wind can substantially contribute to the cooling - for $\theta_{\text {wind }}<\sim 40^{\circ}$ (Figure 2, left). Of course, this limit depends on the other parameters describing the disk and central black hole.

2. The amount of cooling from a disk wind decreases substantially with opening angle, a factor of $\sim 2$ between $\theta_{\text {wind }}=30^{\circ}$ and $\theta_{\text {wind }}=60^{\circ}$ (Figure 2, right).

3. The electron losses in the case of a spherical wind have a different energy dependence than the disk wind losses, so the observed spectra can evolve differently in the two cases, assuming these losses dominate the cooling. We are currently working to quantify the effects of the disk geometry on the observed spectrum.

\section{REFERENCES}

1. Chiang, J. and Mukherjee, R. ApJ, 496, pp. 752-60 (1998)

2. Mücke, A. and Pohl, M. MNRAS, 312, pp. 177-93 (2000)

3. Böttcher, M., Mause, H., and Schlickeiser, R. A\&A, 324, pp. 395-409 (1997)

4. Böttcher, M. and Bloom, S. D. ApJ, 119, pp. 469-477 (2000)

5. Murray, N., Chiang, J., Grossman, S. A., \& Voit, G. M., ApJ, 451, p. 498 (1995) 